\title{
Defined by the Company One Keeps: The Importance of Cardiac Comorbidities after Kidney Transplant Cardiac Remodeling and Outcomes
}

\author{
Dinesh K. Kalra Nidhi Madan \\ Division of Cardiology, Department of Internal Medicine, Rush University Medical Center, Chicago, IL, USA
}

\section{Aesop - \\ "A man is known by the company he keeps"}

Patients with chronic kidney disease (CKD) have an increased risk of cardiovascular disease (CVD) compared with the general population [1]. Especially in those with advanced CKD or on hemodialysis (HD), CV mortality is 10 -fold higher [2]. Many factors account for this, including a high prevalence of risk factors for coronary artery disease (CAD) such as diabetes mellitus (DM), hypertension (HTN), hyperlipidemia, but also other, less appreciated factors such as hemodynamic stressors as volume and pressure overload, anemia, uremic toxins, endothelin, parathormone, alterations in calcium and phosphorus metabolism, and homocysteine [3]. This constellation of cardiac pathology is sometimes termed uremic cardiomyopathy and often includes progressive left ventricular hypertrophy (LVH) and/or systolic dysfunction [4].

Even though kidney transplantation (KT) improves survival rates compared to the patients on $\mathrm{HD}$, the risk of $\mathrm{CV}$ events remains higher in transplant recipients compared with the general population, and $60 \%$ of all deaths are from CV causes [5]. In addition, death from CVD is also the most common cause of graft loss, accounting for $30 \%$ of graft loss overall [6].

There is a strong two-way interplay between CKD and CVD. CV mortality is increased in patients along the entire spectrum of severity of their CKD - in those who are not yet on HD, in those who are on HD, as well as in those who redevelop CKD after successful KT. Similarly, poorer cardiac function worsens renal function and leads to more rapid progression of $\mathrm{CKD}$, which is often clinically recognized as the cardiorenal syndrome [7]. Cardiac adverse remodeling that occurs in both the structural and functional dimensions is an important determinant of this CV mortality, and as such, study of the alterations of LV structure and function prior to and after KT allows longitudinal tracking of those patients who improve and those who remain at high risk for CV complications [8]. LV structural remodeling such as diffuse interstitial fibrosis occurs early in CKD and is associated with myocardial dysfunction [9]. Such changes can be tracked using cardiac MRI (CMR) T1 mapping that can quantify interstitial fibrosis, and also by measuring strain using echocardiography $[9,10]$. Additionally, the development of LVH either by echocardiography or more accurately by

\section{KARGER}

(c) 2020 S. Karger AG, Basel

E-Mail karger@karger.com

www.karger.com/crd
Dinesh K. Kalra, MD, FACC, FSCCT, FSCMR

Division of Cardiology, Department of Internal Medicine Rush University Medical Center

1620 W Harrison St., Kellogg Suite 320, Chicago, IL 60612 (USA)

E-Mail dinesh_kalra@ rush.edu 
CMR increases as renal function deteriorates and predicts future cardiovascular events [11]. Several studies have now shown that cardiac structure and function can also improve after KT - in particular, regression of LVH has been well studied. In a large South Korean study of 767 patients undergoing KT, LVH by transthoracic echocardiography was seen in $71 \%$ and declined to $66 \%$ at 1 year and to $57 \%$ at 5 years after KT. LV geometry also normalized from $20 \%$ at baseline to $22 \%$ at 1 year and to $27 \%$ at 5 years after KT [12]. Longstanding HTN and diabetes are the usual causes of LVH in such patients. $\mathrm{LVH}$ is related not only with worse cardiac but also with worse renal outcomes in this population. Although HD reduces uremic symptoms and improves the quality of life, it may paradoxically exacerbate LVH due to repetitive hemodynamic stresses. In the Frequent Hemodialysis Network Daily Trial, CMR showed less LVH in the group being dialyzed six times per week as compared with the conventional, three-times-per-week group [13]. Reasons for this may include better uremic toxin clearance and better intravascular volume and blood pressure control. In a study of $105 \mathrm{KT}$ recipients [14], LV mass index (LVMI) by echocardiography decreased $13.0 \mathrm{~g} / \mathrm{m}^{2}$ after KT. In another study, An et al. [12] reported similar results showing LVMI improvement of $9.2 \mathrm{~g} / \mathrm{m}^{2}$ at 1 year and $19.1 \mathrm{~g} / \mathrm{m}^{2}$ at 5 years after KT. Along the lines of cardio-renal interaction alluded to above, LVH that does not regress after $\mathrm{KT}$ is associated with renal graft failure. Three pretransplant clinical factors have been identified with lack of LVH regression - these include time on $\mathrm{HD}$, high blood pressure, and anemia.

Aside from these morphological alterations such as LVH discussed above, LV systolic function also plays an important role in cardiorenal outcomes in these patients. In a study that examined the impact of pretransplant LV function on graft survival, an LVEF of $<50 \%$ was associated with poorer graft survival [15]. In another large retrospective echocardiography study of 232 patients, LVEF overall improved from 53 to 56\% [16], and in particular in the 66 patients who had baseline LV systolic dysfunction, it improved from 41 to $50 \%(p<0.001)$. These patients had better CV outcomes than those in whom it did not improve.

Kobayashi et al. [17] demonstrate that the presence of CV comorbidities prevents reverse cardiac remodeling after KT. In their single-center substudy of the prospective DIVAT (computerized and VAlidated data in Transplantation) French database of transplanted patients, they included 56 patients who had echocardiographic data prior to and a median of 24 months after KT. The mean age of the study participants was $48 \pm 15$ years, $73 \%$ were male, and $98 \%$ had HTN at baseline. The mean LVEF improved from $63.9 \%$ prior to KT to $69.6 \%$ after kidney transplantation, whereas there was no significant change in LVMI or in diastolic parameters. In multivariate analysis, valvular heart disease, CAD, heart failure, and DM were associated with no significant or smaller improvement in cardiac structure and function following KT. The limitations of this study are that is a retrospective non-randomized, small study and is subject to bias and also unable define causality. Furthermore, the follow up was short. Nonetheless, the premise that the higher the burden of pre-existing $\mathrm{CV}$ comorbidities, the lesser the cardiac recovery that follows KT makes intuitive sense, and can likely be explained by the degree of myocyte dysfunction that precedes the KT. If cardiac dysfunction has already progressed substantially pre-KT, it may not recover fully and/or take longer time to show recovery than the short time duration that the present study lasted.

The authors should be congratulated for their well-designed and conducted analysis, which adds to the extant literature on this topic. The main message of the present study is that patients with cardiovascular co-morbidities that frequently coexist in such patients, such as CAD, $\mathrm{DM}$, heart failure, or valvular heart disease, had limited favorable cardiac reverse remodeling after KT. This is not unexpected and likely has to do with more extensive cardiomyocyte pathology at baseline when such conditions that cannot be fully captured by metrics, such as LV mass or ejection fracture, coexist. More sensitive imaging techniques to look at myocyte structure and function such as echocardiography and CMR tissue characterization, elastography, diffusion tensor imaging, and multiparametric strain may need to be employed to pick up these changes. As such, this study adds to the literature in confirming the strong interplay that occurs in the cardiorenal axis wherein disease progression in one organ fuels progressive pathology in the other organ and vice versa. Ultimately, for optimal outcomes, we would need to arrest disease progression simultaneously in both organs before this companionship turns sour.

\section{Disclosure Statement}

The authors declare that they have no conflicts of interest to disclose.
78

Cardiology 2020;145:77-79

DOI: $10.1159 / 000505203$
Kalra/Madan 


\section{References}

1 Matsushita K, van der Velde M, Astor BC, Woodward M, Levey AS, de Jong PE, et al.; Chronic Kidney Disease Prognosis Consortium. Association of estimated glomerular filtration rate and albuminuria with all-cause and cardiovascular mortality in general population cohorts: a collaborative meta-analysis. Lancet. 2010 Jun;375(9731):2073-81.

2 Wolfe RA, Ashby VB, Milford EL, Ojo AO, Ettenger RE, Agodoa LY, et al. Comparison of mortality in all patients on dialysis, patients on dialysis awaiting transplantation, and recipients of a first cadaveric transplant. N Engl J Med. 1999 Dec;341(23):1725-30.

3 Meeus F, Kourilsky O, Guerin AP, Gaudry C, Marchais SJ, London GM. Pathophysiology of cardiovascular disease in hemodialysis patients. Kidney Int Suppl. 2000 Aug;76:S140-7.

4 Sood MM, Pauly RP, Rigatto C, Komenda P. Left ventricular dysfunction in the haemodialysis population. NDT Plus. 2008 Aug;1(4): 199-205.

5 Bottomley MJ, Harden PN. Update on the long-term complications of renal transplantation. Br Med Bull. 2013;106(1):117-34.

6 Ojo AO. Cardiovascular complications after renal transplantation and their prevention. Transplantation. 2006 Sep;82(5):603-11.
7 Sarnak MJ, Amann K, Bangalore S, Cavalcante JL, Charytan DM, Craig JC, et al.; Conference Participants. Chronic Kidney Disease and Coronary Artery Disease: JACC State-ofthe-Art Review. J Am Coll Cardiol. 2019 Oct; 74(14):1823-38.

8 Dounousi E, Mitsis M, Naka KK, Pappas C, Lakkas L, Harisis C, et al. Differences in cardiac structure assessed by echocardiography between renal transplant recipients and chronic kidney disease patients. Transplant Proc. 2014 Nov;46(9):3194-8.

9 Hayer MK, Price AM, Liu B, Baig S, Ferro CJ, Townend JN, et al. Diffuse Myocardial Interstitial Fibrosis and Dysfunction in Early Chronic Kidney Disease. Am J Cardiol. 2018 Mar;121(5):656-60.

10 Chiu DY, Green D, Abidin N, Sinha S, Kalra PA. Cardiac imaging in patients with chronic kidney disease. Nat Rev Nephrol. 2015 Apr; 11(4):207-20.

11 Cai QZ, Lu XZ, Lu Y, Wang AY. Longitudinal changes of cardiac structure and function in CKD (CASCADE study). J Am Soc Nephrol. 2014 Jul;25(7):1599-608.

12 An JN, Kim YH, Park JB, Hwang JH, Yoo KD, Park JY, et al. The reciprocal interaction between $\mathrm{LV}$ remodelling and allograft outcomes in kidney transplant recipients. Heart. 2015 Nov;101(22):1826-33.
13 Chan CT, Greene T, Chertow GM, Kliger AS Stokes JB, Beck GJ, et al.; Frequent Hemodialysis Network Trial Group. Effects of frequent hemodialysis on ventricular volumes and left ventricular remodeling. Clin J Am Soc Nephrol. 2013 Dec;8(12):2106-16.

14 Vaidya OU, House JA, Coggins TR, Patil H, Vaidya A, Awad A, et al. Effect of renal transplantation for chronic renal disease on left ventricular mass. Am J Cardiol. 2012 Jul; 110(2):254-7.

15 González Monte E, Mora MT, Polanco N, Morales E, Gutiérrez E, Molina M, et al. Impact of left ventricular dysfunction on renal transplant survival: study of paired kidneys from the same donor. Transplant Proc. 2015 Jan-Feb;47(1):70-2.

16 Hawwa N, Shrestha K, Hammadah M, Yeo PS, Fatica R, Tang WH. Reverse Remodeling and Prognosis Following Kidney Transplantation in Contemporary Patients With Cardiac Dysfunction. J Am Coll Cardiol. 2015 Oct;66(16):1779-87.

17 Kobayashi M, Huttin O, Schikowski J, Bozec E, Zohra L, Frimat L, et al. Cardiovascular Comorbidities Are the Main Predictors of Cardiac Reverse Remodeling following Kidney Transplantation. Cardiology. DOI: 10.1159/ 000504381. 